Richard B. Darst, Department of Mathematics, Colorado State University, Fort Collins, CO 80523, email: darst@@math.colostate.edu

Robert E. Zink, Department of Mathematics, Purdue University, West Lafayette, IN 47907, email: zink@@math.purdue.edu

\title{
A NOTE ON THE DEFINITION OF AN ORLICZ SPACE
}

\begin{abstract}
In the spaces $L_{\mu}^{p}[0,1], p>1$ (which are Orlicz spaces of a very special kind), one has $\|f\|_{p}=\sup \left\{\int_{0}^{1} f g d \mu: \int_{0}^{1}|g|^{q} d \mu \leq 1\right\}=\inf \{t>$ $\left.0: \int_{0}^{1}|f / t|^{p} d \mu \leq 1\right\}=\left(\int_{0}^{1}|f|^{p} d \mu\right)^{1 / p}$, and any one of the three could serve as the definition for $\|f\|_{p}$. For an arbitrary $N$-function, $M$, the analogues of the first two of these formulations yield equivalent norms on the corresponding Orlicz space. On the other hand, the functional $\rho_{M}$, defined on $L_{\mu}^{M}[0,1]$ by $\rho_{M}(f)=M^{-1}\left(\int_{0}^{1} M(f) d \mu\right)$, not only fails to be a norm in most cases, but frequently it is not even a reasonable approximation to the Orlicz norm.
\end{abstract}

\section{In memoriam Władysław Orlicz}

1. Although many characteristics of the $L^{p}$-spaces, $p \geq 1$, are shared by all members of the family of Orlicz spaces, interesting differences between the general and special classes begin to appear very early in the development of the Orlicz theory. One of these differences involves the Orlicz norm on $L_{\mu}^{M}[0,1]$, the definition of which,

$$
\|f\|_{M}=\sup \left\{\int f g d \mu: \int N(g) d \mu \leq 1\right\}
$$

mimics the interplay between $L^{p}$ and $L^{q}$ by employing the $N$-function complementary to $M$. Since the functions $(\cdot)^{p}$ and $(\cdot)^{1 / p}$ are inverses of one another on $\mathbb{R}^{+}$, one's first inclination might be to offer as a candidate for a norm on $L_{\mu}^{M}[0,1]$ the function $\rho_{M}$ given by $\rho_{M}(f)=M^{-1}\left(\int M(f) d \mu\right)$, in analogy

Mathematical Reviews subject classification: Primary: 46E30

Received by the editors April 24, 1995 
with the definition of the $L^{p}$-norm. As Zygmund $[4,170]$ has observed, however, such a proposal is doomed to early rejection because in the general case $\rho_{M}$ is not homogeneous. Indeed, Zaanen [3] has shown that $\rho_{M}$ will be homogeneous only if $M=M(1)(\cdot)^{p}$, for some $p>1$. Nevertheless, one would think that the Orlicz norm and $\rho_{M}$ would be comparable in the sense that there should be positive constants, $K_{1}, K_{2}$, such that $K_{1} \leq \rho_{M} /\|\cdot\|_{M} \leq K_{2}$. The purpose of this note is to show that nothing could be further from the truth.

2. In the sequel, it seems most expedient to employ the terminology and, for the most part, the notation of Krasnosel'skiu and Rutickiu [1].

A continuous, convex function, $M: \mathbb{R} \rightarrow \mathbb{R}^{+}$, is an $N$-function if it is even and if it satisfies both $\lim _{u \rightarrow 0} \frac{M(u)}{u}=0$ and $\lim _{u \rightarrow \infty} \frac{M(u)}{u}=\infty$. Equivalently, $M$ is an $N$-function if and only if there exists a nondecreasing, right-continuous function $p[0,+\infty) \rightarrow \mathbb{R}^{+}$, such that $p(0)=0, p(t)$ is positive for all $t$ in $(0,+\infty), \lim _{t \rightarrow+\infty} p(t)=+\infty$, and $M(u)=\int_{0}^{|u|} p(t) d t, \forall u \in \mathbb{R}$.

If $q:[0,+\infty) \rightarrow \mathbb{R}^{+}$be the right-inverse of $p$; that is, if $q(s)=\sup \{t:$ $p(t) \leq s\}, \forall s \in[0,+\infty)$, then $N: \mathbb{R} \rightarrow \mathbb{R}^{+}$, given by $N(v)=\int_{0}^{|v|} q(s) d s, \forall v \in$ $\mathbb{R}$, is also an $N$-function, and $M$ and $N$ are termed mutually complementary. If $\mu$ be a totally-finite measure on $\mathcal{L}$, the $\sigma$-algebra of Lebesgue-measurable subsets of $[0,1]$, and if $\mathcal{M}$ be the set of all extended-real-valued, measurable $(\mathcal{L})$ functions on $[0,1]$, then the Orlicz class engendered by $\mu$ (and the $N$-function $M)$ is the set

$$
O_{\mu}^{M}[0,1]=\left\{f: f \in \mathcal{M}, \int_{0}^{1} M(f) d \mu<+\infty\right\},
$$

and the corresponding Orlicz space is

$$
L_{\mu}^{M}[0,1]=\left\{f: f \in \mathcal{M}, \int_{0}^{1} f g d \mu<\infty, \forall g \in O_{\mu}^{N}[0,1]\right\}
$$

where $N$ is the $N$-function complementary to $M$. The function $\|\cdot\|_{M \mu}$, defined on $L_{\mu}^{M}[0,1]$ by the relation

$$
\|f\|_{M \mu}=\sup \left\{\int_{0}^{1} f g d \mu: \int_{0}^{1} N(g) d \mu \leq 1\right\}, \quad \forall f \in L_{\mu}^{M}[0,1]
$$

proves to be a norm with respect to which $L_{\mu}^{M}[0,1]$ is a Banach space. From the Young inequality for complementary $N$-functions, $(u v \leq M(u)+N(v)$, $\forall u \in \mathbb{R}, \forall v \in \mathbb{R})$ it follows that $\|f\|_{M \mu} \leq 1+\int_{0}^{1} M(f) d \mu, \forall f \in L_{\mu}^{M}[0,1]$. If, in addition, $\|f\|_{M \mu} \leq 1$, then also $\int_{0}^{1} M(f) d \mu \leq\|f\|_{M \mu}$. 
A sequence $\left\{f_{n}\right\}_{n=1}^{\infty}$ of elements of $L_{\mu}^{M}[0,1]$ is mean convergent to the element $f$ of $L_{\mu}^{M}[0,1]$ iff

$$
\lim _{n} \int_{0}^{1} M\left(f-f_{n}\right) d \mu=0 .
$$

Thus, by virtue of the immediately-preceding inequality, every sequence that converges in the Orlicz norm necessarily will be mean convergent. A meanconvergent sequence need not converge in norm, however.

There are also other norms on $L_{\mu}^{M}[0,1]$ with respect to which this set is transformed into a Banach space. One of these, introduced by Luxemburg (see, for example, [1, 78ff]; see also G. Weiss [2]), is particularly useful, since its definition does not require the use of the function complementary to $M$. This (Luxemburg) norm is given by the relation

$$
\|f\|_{(M) \mu}=\inf \left\{k>0: \int_{0}^{1} M\left(\frac{f}{k}\right) d \mu \leq 1\right\}, \quad \forall f \in L_{\mu}^{M}[0,1] .
$$

One finds that the Orlicz and Luxemburg norms are equivalent; indeed, one has $\|\cdot\|_{(M) \mu} \leq\|\cdot\|_{M \mu} \leq 2\|\cdot\|_{(M) \mu}$.

A set of $N$-functions can be partially ordered in the following manner: if $M_{1}$ and $M_{2}$ are $N$-functions, and if there exist positive constants $u_{0}$ and $k$ such that $M_{1}(u) \leq k M_{2}(u), \forall u \geq u_{0}$, then $M_{1} \prec M_{2}$. If both $M_{1} \prec M_{2}$ and $M_{2} \prec M_{1}$, the sets $L_{\mu}^{M_{1}}[0,1]$ and $L_{\mu}^{M_{2}}[0,1]$ coincide, and the corresponding Orlicz spaces are equivalent.

An $N$-function satisfies the condition $\left(\Delta_{2}\right)$ if there exists a positive $k$ and a nonnegative $u_{0}$ such that $M(2 u) \leq k M(u), \forall u \geq u_{0}$. If $M$ satisfies $\left(\Delta_{2}\right)$, then the associated Orlicz class and Orlicz space coincide. In the absence of this condition, however, one has $O_{\mu}^{M}[0,1] \varsubsetneqq L_{\mu}^{M}[0,1]$.

3. In all that follows, $\mu$ is taken to be the ordinary Lebesgue measure.

Theorem 1 If $M$ is any $N$-function that does not satisfy $\left(\Delta_{2}\right)$, then both

$$
\sup \left\{\rho_{M}(f) /\|f\|_{M}: f \in O_{\mu}^{M}[0,1]\right\}=+\infty,
$$

and

$$
\inf \left\{\rho_{M}(f) /\|f\|_{M}: f \in O_{\mu}^{M}[0,1]\right\}=0 .
$$

Proof. Suppose, first, that $\sup \{\ldots\}<B$. Then $\rho_{M}(f) \leq B\|f\|_{M}, \forall f \in$ $O_{\mu}^{M}[0,1]$. Since $M$ does not satisfy $\left(\Delta_{2}\right)$, there exists a nonnegative element $g$ of $L_{\mu}^{M}[0,1] \backslash O_{\mu}^{M}[0,1]$. For each natural number, $n$, let $g_{n}(t)=\inf \{g(t), n\}, \forall t \in$ 
$[0,1]$. Then each $g_{n}$ belongs to $O_{\mu}^{M}[0,1], g_{1} \leq g_{2} \leq \cdots \leq g$, and $\lim _{n} g_{n}(t)=$ $g(t), \forall t \in[0,1]$. Because the Orlicz norm is monotonic $\lim _{n}\left\|g_{n}\right\|_{M} \leq\|g\|_{M}<$ $+\infty$, and, by virtue of the Lebesgue theorem of monotone convergence,

$$
\lim _{n} \int_{0}^{1} M\left(g_{n}\right) d t=\int_{0}^{1} M(g(t)) d t=+\infty
$$

an impossibility, since

$$
B\|g\|_{M} \geq B\left\|g_{n}\right\|_{M} \geq M^{-1}\left(\int_{0}^{1} M\left(g_{n}\right) d t\right), \quad \forall n .
$$

Now suppose that $\inf \{\ldots\}=b>0$. Let $\left\{h_{n}\right\}_{n=1}^{\infty}$ be a sequence of elements of $O_{\mu}^{M}[0,1]$ such that $\lim _{n} \int_{0}^{1} M\left(h_{n}(t)\right) d t=0$, but $\left\|h_{n}\right\|_{M} \not \rightarrow 0$. (See, for example, $[1,75 \mathrm{ff}]$.$) From \rho_{M}\left(h_{n}\right)>b\left\|h_{n}\right\|_{M}$ follows $M\left(b\left\|h_{n}\right\|_{M}\right)<$ $\int_{0}^{1} M\left(h_{n}(t)\right) d t, \forall n$, so that $\lim _{n} M\left(b\left\|h_{n}\right\|_{M}\right)=0$. But $M^{-1}$ is continuous on $[0,+\infty)$; thus, $\lim _{n}\left\|h_{n}\right\|_{M}=\lim _{n} \frac{1}{b}\left(b\left\|h_{n}\right\|_{M}\right)=0$ !

4. The partial ordering of $N$-functions gives rise to a notion of proximity of the associated Orlicz spaces, in the context of which the following consequence of the theorem is, perhaps, noteworthy.

Since it is possible to construct, for every $p>1$, an $N$-function, $M$, such that $M$ does not satisfy $\left(\Delta_{2}\right)$, and $(\cdot)^{p} \prec M \prec(\cdot)^{p+\varepsilon}, \forall \varepsilon>0$, one sees that an Orlicz space can be "arbitrarily close" to an $L^{p}$-space and yet fail to have its $\rho_{M}$ even remotely comparable to the corresponding norm.

Finally, one is led to question whether this sort of pathology can be associated with $N$-functions that do satisfy the modest growth condition $\left(\Delta_{2}\right)$. Well-known examples supply the answer.

Example 2 For each $\alpha \geq(3+\sqrt{5}) / 2$, the corresponding function $M_{\alpha}: \mathbb{R} \rightarrow$ $\mathbb{R}$, where

$$
M_{\alpha}(u)= \begin{cases}|u|^{\alpha}(|\ln | u||+1) & \text { if } u \neq 0 \\ 0 & \text { if } u=0\end{cases}
$$

is an $N$-function that satisfies a growth condition even stronger than $\left(\Delta_{2}\right)$; viz.,

$$
M_{\alpha}(u v) \leq M_{\alpha}(u) M_{\alpha}(v), \quad \forall u, \quad \forall v .
$$

(For $1<\alpha<(3+\sqrt{5}) / 2, M_{\alpha}$ is not convex on [0,1].) Let $M$ be one of these functions, and let $\varphi:(0,+\infty) \rightarrow \mathbb{R}$ be defined by the relation $\varphi(y)=$ 
$\left(y /\left|\ln \left(y^{1 / \alpha}\right)\right|\right)^{1 / \alpha}, \forall y>0$. Let $K$ be a real number, greater than $e$, such that $\ln (\ln x+1)^{1 / \alpha}>1, \forall x>K$. Then, for all such $x$,

$$
\begin{aligned}
\varphi\left(x^{\alpha}(\ln x+1)\right) & =\frac{x(\ln x+1)^{1 / \alpha}}{\left(\ln \left[x(\ln x+1)^{1 / \alpha}\right]\right)^{1 / \alpha}} \\
& =\frac{x(\ln x+1)^{1 / \alpha}}{\left.\left[\ln x+\ln (\ln x+1)^{1 / \alpha}\right]\right]^{1 / \alpha}} \\
& <x<2 \varphi\left(x^{\alpha}(\ln x+1)\right) ;
\end{aligned}
$$

thus,

$$
\varphi(y)<M^{-1}(y)<2 \varphi(y), \quad \forall y>y_{0}=K^{\alpha}(\ln K+1) .
$$

Let $n$ be any natural number such that $r=\exp \left(\alpha(16 n)^{\alpha}\right)>y_{0}$; let $E$ be a measurable subset of $[0,1]$ such that $\mu(E)=1 / r$; let $t=r y_{0}$; let $f=t \chi_{E} ;$ and let $b=\|f\|_{(M)} / M^{-1}\left(\int M(f) d \mu\right)$. Since $M$ satisfies $\left(\Delta_{2}\right)$, $\int M\left(\frac{f}{\|f\|_{(M)}}\right) d \mu=1$, and

$$
\mu(E) M\left(t / b M^{-1}\left(\int M(f) d \mu\right)\right)=1 .
$$

Thus,

$$
\begin{aligned}
t / b & =M^{-1}(M(t) \mu(E)) M^{-1}(1 / \mu(E)) \\
& <4\left[\frac{M(t) \mu(E)}{\ln (M(t) \mu(E))^{1 / \alpha}}\right]^{1 / \alpha}\left[\frac{1 / \mu(E)}{\ln (1 / \mu(E))^{1 / \alpha}}\right]^{1 / \alpha} \\
& =\frac{4(M(t))^{1 / \alpha}}{\left[\ln (M(t) \mu(E))^{1 / \alpha}\right]^{1 / \alpha}\left[\ln (1 / \mu(E))^{1 / \alpha}\right]^{1 / \alpha}} \\
& <\frac{4 t(\ln t+1)^{1 / \alpha}}{\left[\ln \left(2 \mu(E) t^{\alpha}\right)^{1 / \alpha}\right]^{1 / \alpha}(16 n)} \\
& <\frac{\left(2^{1 / \alpha}\right) t(\ln t)^{1 / \alpha}}{4 n\left[\ln \left(2 \mu(E) t^{\alpha / 2}\right)^{1 / \alpha}+\ln t^{1 / 2}\right]^{1 / \alpha}} \\
& <\frac{\left(2^{1 / \alpha}\right)(\ln t)^{1 / \alpha} t}{4 n\left(2^{-1 / \alpha}\right)(\ln t)^{1 / \alpha}}<\frac{t}{n},
\end{aligned}
$$

so that

$$
b=\|f\|_{(M)} / M^{-1}\left(\int M(f) d \mu\right)>n .
$$

Since $n$ may be taken to be arbitrarily large, it follows that

$$
\sup \left\{\|f\|_{M} / M^{-1}\left(\int M(f) d \mu\right): f \in O_{\mu}^{M}[0,1]\right\}=+\infty \text {. }
$$


Example 3 Let $M$ be the $N$-function defined, for all real $u$, by the relation $M(u)=u^{2} / \ln (e+|u|)$, and let $\psi(y)=y^{1 / 2}\left[\ln \left(y^{1 / 2}\right)\right]^{1 / 2}, \forall y>1$. Then

$$
\psi\left(x^{2} / \ln (e+x)\right)=\frac{x}{[\ln (e+x)]^{1 / 2}}\left[\ln \left(\frac{x}{[\ln (e+x)]^{1 / 2}}\right)\right]^{1 / 2}<x, \quad \forall x>e,
$$

and

$$
\begin{aligned}
\psi\left(x^{2} / \ln (e+x)\right) & =\frac{x}{[\ln (e+x)]^{1 / 2}}\left[\ln \left(\frac{x^{1 / 2}}{[\ln (e+x)]^{1 / 2}}\right)+\ln x^{1 / 2}\right]^{1 / 2} \\
& >x\left[\frac{\ln x^{1 / 2}}{\ln (e+x)}\right]^{1 / 2} \\
& >x / 2, \quad \forall x>e .
\end{aligned}
$$

Thus, $\psi(y)<M^{-1}(y)<2 \psi(y), \forall y>e^{2} / \ln 2 e$.

Let $n$ be a natural number, and let $E$ be a measurable subset of $[0,1]$ for which $\mu(E)=\exp \left(-8 n^{2}\right)$. Choose $t$ such that $t>\exp \left(8 n^{2}\right) \ln (e+t)$; let $f=t \chi_{E}$; and let $b=\|f\|_{(M)} / M^{-1}\left(\int M(f) d \mu\right)$. Since $M$ satisfies $\left(\Delta_{2}\right)$, one has

$$
1=\int M\left(f /\|f\|_{(M)}\right) d \mu=M\left(t /\|f\|_{(M)}\right) \mu(E),
$$

so that

$$
\begin{aligned}
t / b & =M^{-1}(M(t) \mu(E)) M^{-1}(1 / \mu(E)) \\
& >[M(t) \mu(E)]^{1 / 2}\left[\ln (M(t) \mu(E))^{1 / 2}\right]^{1 / 2}[1 / \mu(E)]^{1 / 2}\left[\ln (1 / \mu(E))^{1 / 2}\right]^{1 / 2} \\
& =t /[\ln (e+t)]^{1 / 2}\left[\ln \left(\frac{t^{2} \mu(E)}{\ln (e+t)}\right)^{1 / 2}\right]^{1 / 2}\left[\ln (1 / \mu(E))^{1 / 2}\right]^{1 / 2}
\end{aligned}
$$

Thus,

$$
\begin{aligned}
1 / b^{2} & >[1 / \ln (e+t)]\left[\ln (t \mu(E) / \ln (e+t))^{1 / 2}+\ln t^{1 / 2}\right]\left[\ln (1 / \mu(E))^{1 / 2}\right] \\
& >[1 / \ln (e+t)]\left[\ln t^{1 / 2}\right]\left[\ln (1 / \mu(E))^{1 / 2}\right] \\
& =(1 / 4)[\ln t / \ln (e+t)] \ln (1 / \mu(E)) \\
& >(1 / 8) \ln (1 / \mu(E))=n^{2}
\end{aligned}
$$

so that $b<1 / n$.

It follows that $\inf \left\{\|f\|_{M} / \rho_{M}(f): f \in O_{\mu}^{M}[0,1]\right\}=0$.

$\mathrm{Ah}$, Horatio, there are stranger things in heaven and earth ... 


\section{References}

[1] M. A. Krasnosel'skiı̌ and Ya. B. Rutickiı̌, Convex Functions and Orlicz Spaces, Noordhoff, Groningen, 1961.

[2] G. Weiss, A note on Orlicz spaces, Portugaliae Math, 15 (1956), 35-47.

[3] A. C. Zaanen, Some remarks about the definition of an Orlicz space, Measure Theory Oberwolfach 1981, Lecture Notes in Maths, 945 (1982), 261-268, Springer-Verlag, Berlin.

[4] A. Zygmund, Trigonometric Series, Vol. I, Cambridge University Press, New York, 1959. 